\title{
ANGLES AND THE INNER RADIUS OF UNIVALENCY
}

\author{
MATTI LEHTINEN
}

\section{Introduction}

Let $A$ be a quasidisc. Denote by $\varrho_{A}$ the density of the Poincaré metric in $A$, so normalized that $\varrho_{H}(x+i y)=1 /(2 y)$ for the upper half-plane $H$. The inner radius of univalency $\sigma_{I}(A)$ of $A$, introduced by Lehto [6], is the supremum of numbers $a$ such that every locally injective meromorphic function $f$ defined in $A$, which satisfies $\left\|S_{f}\right\|_{A} \leqq a$, is univalent. Here $S_{f}$ stands for the Schwarzian derivative of $f$ and the norm $\left\|S_{f}\right\|_{A}$ is the supremum of the numbers $\left|\mathrm{S}_{f}(z)\right| / \varrho_{A}(z)^{2}$ for $z$ in $A$. It follows from the definition and the transformation properties of the Schwarzian that the inner radius of univalency is invariant under Möbius transformations. It can be shown that $\sigma_{I}(A)$ equals the infimum of $\left\|S_{f}\right\|_{A}$ for those conformal maps $f$ of $A$ for which $f(A)$ is not a quasidisc.

It is known that $0<\sigma_{I}(A) \leqq 2$ for every quasidisc $A$ and $\sigma_{I}(A)=2$ if and only if $A$ is a disc or a half-plane. The only other cases in which the inner radii of univalency are known exactly are those of an angular domain $A=A_{k}=\{z|| \arg z \mid<k \pi / 2\}$, $0<k<2$; for it

$$
\sigma_{I}\left(A_{k}\right)=2 k(1-|1-k|),
$$

[5, 2], and the one in which $A$ is a domain bounded by a branch of a hyperbola [4]. Upper and lower estimates, based on (1), have been obtained for $\sigma_{I}(A)$ when $A$ is one of the domains bounded by an ellipse [6,3,4]. In this paper, (1) together with elementary geometrical considerations is further utilized in order to determine $\sigma_{I}(A)$ when $A$ is a domain bounded by a triangle or a regular $n$-gon, $n \geqq 3$. An upper estimate of the inner radius of univalency for general domains bounded by a curve possessing an angle is given in Section 4.

For any plane domain $A$ we denote by $A^{*}$ the complementary domain $\bar{C} \backslash A$. 


\section{A majorization principle}

We estimate $\sigma_{I}(A)$ by comparing $A$ to angular domains in the interior or exterior of $A$. The general procedure is described by the following two lemmas.

Lemma 1. Let $A$ be a quasidisc and let $A \subset B_{k}$ where $B_{k}$ is a domain Möbius equivalent to $A_{k}$ for some $k$. If $0<k<1$ and a vertex $v$ of $B_{k}$ is on $\partial A$, then $\sigma_{I}(A) \leqq 2 k^{2}$. If $1<k<2$ and there exist points $z_{1}, z_{2}$ in $A$ and a Möbius transformation $g$ satisfying $g\left(B_{k}\right)=A_{k}$ and $g\left(z_{1}\right)=e^{i k \pi / 2}, g\left(z_{2}\right)=e^{-i k \pi / 2}$, then $\sigma_{I}(A) \leqq 4 k-2 k^{2}$.

Proof. In the case $0<k<1$ we let $g, g(v)=0$, be a Möbius transformation carrying $B_{k}$ onto $A_{k}$. Set $f(z)=\log g(z)$. Then, by the quasicircle criterion of Ahlfors, $f(A)$ is no quasidisc, and $\sigma_{I}(A) \leqq\left\|S_{f}\right\|_{A}$. But, by the Schwarz lemma, $\sigma_{A}(z) \geqq \varrho_{B_{k}}(z)$ for $z \in A$, whence $\left\|S_{f}\right\|_{A} \leqq\left\|S_{f}\right\|_{B_{k}}=2 k^{2}$. In the second case, there exists a conformal $f: A_{k} \rightarrow \bar{C}$ such that $\left\|S_{f}\right\|_{A_{k}}=4 k-2 k^{2}$ and $f\left(e^{i k \pi / 2}\right)=f\left(e^{-i k \pi / 2}\right)=\infty$ [2]. Then $f(g(A))$ is not a Jordan domain, and a reasoning similar to the one above shows that $\sigma_{I}(A) \leqq\left\|S_{f}\right\|_{A}$.

Lem ma 2. Let $A$ be a quasidisc. If each two-element subset of $A$ is contained in the closure of a quasidisc $B \subset A$ such that $\sigma_{I}(B) \geqq m$, then $\sigma_{I}(A) \geqq m$.

Proof. Let an $\varepsilon>0$ be given. By definition, a locally injective $f: A \rightarrow C$ exists such that $\left\|S_{f}\right\|_{A}<\sigma_{I}(A)+\varepsilon$ and $f$ is not univalent. Then $f(z)=f(w)$ for some $z \neq w$. There is a quasidisc $B$ in $A$, such that $\{z, w\} \subset B$ and $\sigma_{I}(B) \geqq m$. Since either $f$ is not univalent in $B$ or $f(B)$ is not a quasidisc, $\left\|S_{f}\right\|_{B} \geqq \sigma_{I}(B)$. The monotony of the Poincaré metric again implies that $\left\|S_{f}\right\|_{A} \geqq\left\|S_{f}\right\|_{B}$. Hence $\sigma_{I}(A)>m-\varepsilon$, and the assertion follows.

\section{Domains bounded by a triangle or a regular $n$-gon}

Lemmas 1 and 2 combined with elementary geometrical considerations yield almost immediately

Theorem 1. If $T$ is the finite domain bounded by a triangle with smallest angle $k \pi$, then $\sigma_{I}(T)=2 k^{2}$ and $\sigma_{I}\left(T^{*}\right)=4 k-2 k^{2}$.

Proof. That $\sigma_{I}(T) \leqq 2 k^{2}$ is an immediate consequence of Lemma 1. In the opposite direction, one observes that any two points $z_{1}, z_{2}$ in $T$ are on the boundary of a triangle $T^{\prime}$ similar to $T$ and lying inside $T$ in such a way that one of the points $z_{1}, z_{2}$ is a vertex of $T^{\prime}$. It is easy to convince oneself that a vertex and a point on the perimeter of a triangle can be joined in the triangle by two circular arcs (or an arc and a part of a side of the triangle) meeting at an angle at least as large as the smallest angle in the triangle. The desired conclusion follows from Lemma 2. 
To prove the equality for $\sigma_{I}\left(T^{*}\right)$, we first observe that each pair of points in $T^{*}$ is in one of the three angular domains determined by the exteriors of the angles of $\partial T$. Hence $\sigma_{I}\left(T^{*}\right) \geqq 4(2-k)-2(2-k)^{2}=4 k-2 k^{2}$. On the other hand, for each $k^{\prime}>k$ one can draw two symmetric circular arcs in $T$ meeting at the angle $k^{\prime} \pi$ and tangent to $\partial T$ at two points equidistant from the common points of the arcs. By Lemma 1, then, $\sigma_{I}\left(T^{*}\right) \leqq 4 k^{\prime}-2 k^{\prime 2}$, and the assertion follows.

By a similar argument one proves also

The o r e 2 2. Let $P_{n}$ be the finite domain bounded by a regular $n$-gon. Then $\sigma_{I}\left(P_{n}\right)=$ $2((n-2) / n)^{2}$ and $\sigma_{I}\left(P_{n}^{*}\right)=2-8 / n^{2}$.

Proof. Upper estimates for both $\sigma_{I}\left(P_{n}\right)$ and $\sigma_{I}\left(P_{n}^{*}\right)$ are obtained exactly as in the proof of Theorem 1.

For a lower estimate of $\sigma_{I}\left(P_{n}\right)$, consider two points $z_{1}$ and $z_{2}$ in $P_{n}$. Let the line through these points intersect $\partial P_{n}$ at $w_{1}$ and $w_{2}$. Observe that an appropriate homothety or translation applied to the circle inscribed in $P_{n}$ shows that for any two vertices of $\partial P_{n}$ there exist two circular arcs in $P_{n}$ (or an arc and a side of $P_{n}$ ) joining the vertices and meeting each other in the angle $(1-2 / n) \pi$. Assume first that $w_{1}$ is a vertex and that $w_{2}$ lies on a side whose endpoints are $v_{1}$ and $v_{2}$. Homotheties with center $w_{1}$ transform arcs joining $w_{1}$ to $v_{1}$ and $v_{2}$ into arcs joining $w_{1}$ to $w_{2}$ in $P_{n}$ preserving the angles at $w_{1}$. Finally, let also $w_{1}$ be an interior point of a side, with endpoints $v_{3}$ and $v_{4}$. We can now join $w_{2}$ to $v_{3}$ and $v_{4}$ by pairs of arcs meeting at the angle $(1-2 / n) \pi$. The arcs can be shown to be pairwise tangent to each other at $w_{2}$, and thus an anglepreserving homothety with center $w_{2}$ can be performed in order to obtain arcs joining $w_{2}$ to $w_{1}$ in $P_{n}$. By Lemma $2, \sigma_{I}\left(P_{n}\right) \geqq 2(1-2 / n)^{2}$.

For $P_{n}^{*}$, one observes that any two points in the domain are either in a half-plane contained in the domain or (as can be seen by applying two consequtive homotheties to the figure) on the boundary of a regular $n$-gon which contains $P_{n}$. We may thus consider only pairs of points $w_{1}, w_{2}$ on $\partial P_{n}$. Assume that $w_{1}$ is a vertex and $w_{2}$ is on a side with endpoints $v_{1}$ and $v_{2}$. Circular arcs through $w_{2}, v_{1}, w_{1}$ and $w_{2}, v_{2}, w_{1}$, respectively, meet the circle circumscribed around $P_{n}$ at two points each and are contained in $P_{n}^{*}$. The larger angle between them is at most $(1+2 / n) \pi$, as can be seen by comparing it to the angle between the circumscribed circle and the sides of $\partial P_{n}$. If $w_{1}$ is on a side with endpoints $v_{3}$ and $v_{4}$, an arc joining $w_{2}, v_{2}, w_{1}$ in $P_{n}^{*}$ is found between the arcs joining $w_{2}, v_{2}, v_{3}$ and $w_{2}, v_{2}, v_{4}$. Similarly, an arc joining $w_{2}, v_{1}$ and $w_{1}$ is found; these arcs meet again with larger angle at most $(1+2 / n) \pi$. The estimate follows from Lemma 2.

Remark. The results in Theorems 1 and 2, in the case of a finite domain, have been obtained also by D. Calvis [1]. 


\section{An upper estimate for domains with a boundary angle}

The majorization principle incorporated in Lemma 1 immediately yields an upper estimate for $\sigma_{I}(A)$ in the case of an angle less than $\pi$ in $\partial A$. For our purposes the following definition of a boundary angle is appropriate: we say that $\partial A$ possesses an angle $k \pi$ at $z_{0}$ if for every $\varepsilon \in(0, \min \{\mathrm{k}, 2-k\})$ there exists a Möbius transformation $g_{\varepsilon}$ such that $g_{\varepsilon}\left(z_{0}\right)=\infty$ and

$$
A_{k-\varepsilon} \cap D^{*} \subset g_{\varepsilon}(A) \cap D^{*} \subset A_{k+\varepsilon} \cap D^{*},
$$

where $D$ is the unit disc.

Theorem 3. [3] Assume $A$ has a boundary angle $k \pi, 0<k<1$, at a boundary point $z_{0}$. Then $\sigma_{I}(A) \leqq 2 k^{2}$.

Proof. Assume $0<\varepsilon<1-k$. Without loss of generality, assume $z_{0}=0, A \cap D \subset$ $A_{k+\varepsilon} \cap D$. There is a Möbius transformation $g$ such that $g(A) \subset A_{k+\varepsilon}, g(0)=0$. By Lemma $1, \sigma_{I}(g(A))=\sigma_{I}(A) \leqq 2(k+\varepsilon)^{2}$.

For a corresponding result concerning boundary angles exceeding $\pi$, it is useful to recall a few facts concerning the universal Teichmüller space $T$ (see e.g. [5]). We let $T$ be the space whose points are equivalence classes of complex dilatations $\mu$ defined in $\boldsymbol{C}$, zero in $H^{*}$, the equivalence of $\mu$ and $v$ being defined by $f_{\mu}\left|\boldsymbol{R}=f_{v}\right| \boldsymbol{R}$, where $f_{\mu}$ is a quasiconformal mapping of the plane with complex dilatation $\mu$, normalized at three points on the real axis. The distance of $\mu$ and $v$ is the minimum of numbers $(1 / 2) \log K_{f_{\mu} \circ f_{v}}-1$. The space $T(1)$ of $S_{f}$, where $f$ is conformal in $H^{*}$ and maps $H^{*}$ onto a quasidisc, endowed with the norm $\left\|S_{f}\right\|_{H^{*}}$, is homeomorphic to $T$; the homeomorphism $\Phi$ is the one that attaches $S_{f_{\mu}}$ to the equivalence class of $\mu$. If $f$ is a conformal map of the lower half-plane onto $A$, the inner radius of univalency of $A$ is the distance of $S_{f}$ from the boundary of $T(1)$.

Lemma 3. Let $A$ be a quasidisc. If $c<\sigma_{I}(A)$ and $f$ is a conformal map of $A$ such that $\left\|S_{f}\right\|_{A} \leqq c$, then $f(A)$ is a $K$-quasidisc, where $K$ is bounded by a constant depending on $A$ and $c$ only.

Proof. Let $g: H^{*} \rightarrow A$ be conformal. By the transformation rules of the Schwarzian, $\left\|S_{f}\right\|_{A}=\left\|S_{f \circ g}-S_{g}\right\|_{H^{*}}$. The preimage under $\Phi$ of the compact $c$-neighborhood of $S_{g}$ is compact in $T$. It follows that $f \circ g$ has a $K$-quasiconformal extension to $H$ with $K$ bounded by a constant depending on $A$ and $c$. Hence $f(A)$ is a $K$-quasidisc.

To prove the upper estimate for $\sigma_{I}(A)$ for domains having an angle larger than $\pi$ in the boundary, we still need the following observation. For each $k \in(1,2)$, let $E_{k}$ be the domain $A_{k} \cap\left\{z \mid 1-z \in A_{k}\right\}$ and $f_{k}$ the conformal map of $A_{k}$ onto $E_{k}$ taking 0 to 1 , infinity to 0 and $e^{ \pm i k \pi / 2}$ to infinity [2]. Then $f_{k}$ maps the arc of radius 1 and center 0 onto the imaginary axis.

Theorem 4. Let $A$ be a quasidisc possessing at $z_{0} \in \partial A$ the angle $k \pi, 1<k<2$. Then $\sigma_{I}(A) \leqq 4 k-2 k^{2}$. 
Proof. Assume that $\sigma_{I}(A)>4 k-2 k^{2}$. Let $\left(k_{n}\right)$ be a decreasing sequence tending to $k$. By a suitable Möbius transformation we may arrange $A$ to lie in $A_{k_{n}}$ in such a way that there are points in $A$ closer to the points $e^{ \pm i k_{n} \pi / 2}$ than, say, $2\left(k_{n}-k\right)$. Then the closure of $f_{n}(A)$ contains points $0, i y_{n},-i y_{n}$ and $x, 0<x \leqq 1$, and $y_{n}$ tends to infinity together with $n$. Clearly, $f_{n}(A)$ cannot be a $K$-quasidisc with a fixed $K$ for all $n$. On the other hand, $\left\|S_{f}\right\|_{A} \leqq\left\|S_{f_{n}}\right\|_{A_{n}}=4 k_{n}-2 k_{n}^{2} \leqq c<\sigma_{I}(A)$ for $n$ large enough. This is a contradiction with Lemma 3 .

Of course, estimates of $\sigma_{I}(A)$ based on local properties of $\partial A$ are in general not very sharp. Let, for example, $A$ be the exterior of a rectangle with sides 1 and $a>1$. By Theorem $4, \sigma_{I}(A) \leqq 3 / 2$. On the other hand, one can inscribe in $\partial A$ two symmetric arcs which meet at the angle $k \pi=2 \pi-2 \arctan (1 / a)$. By Lemma $1, \sigma_{I}(A) \leqq 4 k-2 k^{2}$, and $\sigma_{I}(A)$ tends to 0 as $a$ grows to infinity.

\title{
References
}

[1] CALvis, D.: The inner radius of univalence of normal circular triangles and regular polygons. Complex Variables Theory Appl. 4, 1985, 295-304.

[2] Lehtinen, M.: On the inner radius of univalency for noncircular domains. - Ann. Acad. Sci. Fenn. Ser. A 1 Math. 5, 1980, 45-47.

[3] Lehtinen, M.: Remarks on the inner radius of univalency of quasidiscs. - Proc. of the Second Finnish-Polish Summer School in Complex Analysis at Jyväskylä, University of Jyväskylä, 1984, 73-78.

[4] LeHTINEN, M.: Estimates of the inner radius of univalency of domains bounded by conic sections. Ann. Acad. Sci. Fenn. Ser. A I Math. 10, 1985, 349-353.

[5] Lehro, O.: Univalent functions and Teichmüller theory. - Proc. of the First Finnish-Polish Summer School in Complex Analysis at Podlesice, University of Lodz, 1977, 11-33.

[6] LeHto, O.: Remarks on Nehari's theorem about the Schwarzian derivative and schlicht functions. - J. Analyse Math. 36, 1979, 184-190.

\author{
University of Helsinki \\ Department of Mathematics \\ SF-00100 Helsinki
}

Finland 